\title{
Numerical Solution of the \\ Inverse Problem of Determining an Unknown Source Term in a Heat Equation
}

\author{
Xiuming Li and Suping Qian \\ Department of Mathematics, Changshu Institute of Technology, Changshu, Jiangsu 215500, China \\ Correspondence should be addressed to Xiuming Li, lixiumingxu@gmail.com
}

Received 8 March 2012; Accepted 7 May 2012

Academic Editor: Hui-Shen Shen

Copyright (c) 2012 X. Li and S. Qian. This is an open access article distributed under the Creative Commons Attribution License, which permits unrestricted use, distribution, and reproduction in any medium, provided the original work is properly cited.

This paper investigates the inverse problem of determining a heat source in the parabolic heat equation using the usual conditions. Firstly, the problem is reduced to an equivalent problem which is easy to handle using variational iteration method. Secondly, variational iteration method is used to solve the reduced problem. Using this method a rapid convergent sequence can be produced which tends to the exact solution of the problem. Furthermore, variational iteration method does not require the discretization of the problem. Two numerical examples are presented to illustrate the strength of the method.

\section{Introduction}

In the process of transportation, diffusion, and conduction of natural materials, the following heat equation is induced:

$$
u_{t}-a^{2} \Delta u=f(x, t ; u), \quad(x, t) \in \Omega \times(0, T]
$$

where $u$ represents state variable, $a$ is the diffusion coefficient, $\Omega$ is a bounded domain in $R^{d}$, and $f$ denotes physical laws, which means source terms here. There are many researches on such inverse problems of determining source terms from 1970s, since the characteristics of sources in practical problems are always unknown. And the inverse problems are unstable in nature from indirect observable data which contain measurement errors. The major difficulty in establishing any numerical algorithm for approximating the solution is the ill-posedness of the problem and the ill-conditioning of the resultant discretized matrix. The inverse problem 
of determining an unknown heat-source function in the heat conduction equation has been considered in many papers [1-8]. For $f=f(u)$, the inverse source problem with additional data was studied by Cannon, Duchateau, and Fatullayev [1,2]. In [3, 4], the source is sought as a function of both space and time but is additive or separable. However, in all the other studies the source has been sought as a function of space or time only [5-8].

In this paper, the heat source is taken to be time dependent only, and the overdetermination is the transient temperature measurement recorded by a single thermocouple installed in the interior of the heat conductor. These measurements ensure that the inverse problem has a unique solution, but this solution is unstable; hence the problem is illposed. The inverse problems are formulated in Section 2. Several numerical methods have been proposed for the inverse source problem [5-12]. In this work, we extend the use of variational iteration method (VIM) to this inverse source problem. The VIM was proposed originally by He $[13,14]$. This method is based on the use of Lagrange multipliers for identification of optimal values of parameters in a functional. This method gives rapidly convergent successive approximations of the exact solution if such a solution exists. For concrete problems, a few number of approximations can be used for numerical purposes with a high degree of accuracy. Furthermore, VIM does not require the discretization of the problem. Thus the variational iteration method is suitable for finding the approximation of the solution without discretization of the problem. It was successfully applied to two-point boundary value problems, partial differential equations, evolution equations, and other fields [13-26].

The rest of the paper is organized as follows. In the next section, we formulate the problem mathematically. The VIM is introduced and applied to the inverse source problem in Section 3. The numerical examples are present in Section 4. Section 5 ends this paper with a brief conclusion.

\section{Formulation of the Inverse Problem}

Let $T>0, \alpha \in(0,1)$ be fixed numbers, and let us consider first the one-dimensional timedependent problem in which the source $f(x, t ; u)=f(t)$ depends on time only.

Find the temperature $u \in H^{2+\alpha, 1+\alpha / 2}([0,1] \times[0, T])$ and the heat source $f \in H^{\alpha / 2}([0, T])$ which satisfy the heat-conduction equation with a time-dependent source, namely,

$$
u_{t}=a^{2} u_{x x}+f(t), \quad(x, t) \in(0,1) \times(0, T],
$$

subject to the initial and boundary conditions

$$
\begin{gathered}
u(x, 0)=u_{0}(x), \quad 0 \leq x \leq 1, \\
u(0, t)=g_{0}(t), \quad u(1, t)=g_{1}(t), 0 \leq t \leq T
\end{gathered}
$$

and the overspecified condition

$$
u\left(x_{0}, t\right)=h(t), \quad 0 \leq t \leq T,
$$


where $x_{0} \in(0,1)$ is the interior location of a thermocouple recording the temperature measurement, $u_{0}(x), g_{0}(t), g_{1}(t), h(t)$ are given functions satisfying the compatibility conditions

$$
u_{0}(0)=g_{0}(0), \quad u_{0}(1)=g_{1}(0), \quad u_{0}\left(x_{0}\right)=h(0),
$$

and the functions $u(x, t)$ and $f(t)$ are unknown.

From [5], we know that, if $g_{0}, g_{1}, h \in H^{1+\alpha / 2}([0, T]), u_{0} \in H^{2+\alpha}([0,1])$ and conditions (2.2)-(2.4) are consistent up to the first order, then problem (2.1)-(2.4) has a unique solution.

The model problem presented here used to describe a heat transfer process with a time-dependent source produces the temperature at a given point $x_{0}$ in the spatial domain at time $t$. Thus, the purpose of solving this inverse problem can be viewed as an inverse control problem to identify the source control parameter that produces at any given time a desired temperature at a given point $x_{0}$ in the spatial domain.

Although sufficient conditions for the solvability of the problem are provided [5], problem (2.1)-(2.5) is still ill-posed since small errors, inherently present in any practical measurement, give rise to unbounded and highly oscillatory solutions. We will change (2.1) to an equation with one unknown function which is easy to handle using VIM.

Using the two following transformations:

$$
r(t)=\int_{0}^{t} f(\xi) d \xi, \quad v(x, t)=u(x, t)-r(t)
$$

Equation (2.1) is transformed into the following equation:

$$
v_{t}=a^{2} v_{x x}, \quad(x, t) \in(0,1) \times(0, T],
$$

with the initial and boundary conditions

$$
\begin{gathered}
v(x, 0)=u_{0}(x), \quad 0 \leq x \leq 1, \\
v(0, t)=g_{0}(t)-r(t), \quad v(1, t)=g_{1}(t)-r(t), \quad 0 \leq t \leq T
\end{gathered}
$$

and the overspecification at a point $x_{0}$ :

$$
v\left(x_{0}, t\right)=h(t)-r(t), \quad 0 \leq t \leq T .
$$

\section{Analysis and Application of He's Variational Iteration Method}

Consider the differential equation

$$
L u+N u=g(x)
$$


where $L$ and $N$ are linear and nonlinear operators, respectively, and $g(x)$ is the source inhomogeneous term. In [9-15], the VIM was introduced by He where a correct functional for (3.1) can be written as

$$
u_{n+1}(x)=u_{n}(x)+\int_{0}^{x} \lambda\left\{L u_{n}(t)+N \tilde{u}_{n}(t)-g(t)\right\} d t
$$

where $\lambda$ is a general Lagrangian multiplier [13], which can be identified optimally via variational theory, and $\tilde{u}_{n}$ is a restricted variation which means $\delta \tilde{u}_{n}=0$. By this method, it is required first to determine the Lagrangian multiplier $\lambda$ that will be identified optimally. The successive approximates $u_{n+1}, n \geq 0$, of the solution $u$ will be readily obtained upon using the determined Lagrangian multiplier and any selective function $u_{0}$. Consequently, the solution is given by

$$
u=\lim _{n \rightarrow \infty} u_{n}
$$

The variational iteration method has been shown to solve easily and accurately a large class of problems with approximations converging rapidly to accurate solutions.

For variational iteration method, the key is the identification of Lagrangian multiplier. For linear source, their exact solutions can be obtained by only one iteration step due to the fact that the Lagrangian multiplier can be identified exactly. For nonlinear source, the lagrange multiplier is difficult to be identified exactly. To overcome the difficulty, we apply restricted variations to nonlinear term. Due to the approximate identification of the Lagrangian multiplier, the approximate solutions converge to their exact solutions relatively slowly. It should be specially pointed out that the more accurate the identification of the multiplier, the faster the approximations converge to their exact solutions.

For (2.7), according to the VIM, we consider its correct functional in $t$-direction in the following form:

$$
v_{n+1}(x, t)=v_{n}(x, t)+\int_{0}^{t} \lambda\left\{\frac{\partial v_{n}(x, s)}{\partial s}-a^{2} \frac{\partial^{2} \widetilde{v}_{n}(x, s)}{\partial x^{2}}\right\} d s
$$

where $\lambda$ is the general Lagrangian multiplier, which can be identified optimally via variational theory, and $\widetilde{v}_{n}$ is a restricted variation which means $\delta \widetilde{v}_{n}=0$.

To find the optimal value of $\lambda$, we have

$$
\delta v_{n+1}(x, t)=\delta v_{n}(x, t)+\delta \int_{0}^{t} \lambda\left\{\frac{\partial v_{n}(x, s)}{\partial s}-a^{2} \frac{\partial^{2} \widetilde{v}_{n}(x, s)}{\partial x^{2}}\right\} d s=0
$$

that results

$$
\delta v_{n+1}(x, t)=\delta v_{n}(x, t)(1+\lambda)-\int_{0}^{t} \delta v_{n}(x, s) \lambda^{\prime} d s=0
$$


which yields

$$
\begin{gathered}
\lambda^{\prime}(t)=0, \\
1+\lambda(t)=0 .
\end{gathered}
$$

Thus we have

$$
\lambda(t)=-1,
$$

and therefore, we have the following iteration formula:

$$
v_{n+1}(x, t)=v_{n}(x, t)-\int_{0}^{t}\left\{\frac{\partial v_{n}(x, s)}{\partial s}-a^{2} \frac{\partial^{2} v_{n}(x, s)}{\partial x^{2}}\right\} d s .
$$

Now taking $v_{0}(x, t)=v(x, 0)=u_{0}(x)$ as an initial value and using (3.9), we can obtain the solution of (2.7) as a convergent sequence. The solution of (2.1) is obtained in the following form:

$$
\begin{gathered}
f(t)=\left(g_{0}(t)-v(0, t)\right)^{\prime}, \\
u(x, t)=v(x, t)+r(t)=v(x, t)+g_{0}(t)-v(0, t) .
\end{gathered}
$$

\section{Numerical Examples}

In this section, we present and discuss the numerical results by employing VIM for two test examples. For these examples, we have taken $a=1$ and $T=1$. Results demonstrate present method is remarkably effective.

Example 4.1. With the input data

$$
\begin{gathered}
u(x, 0)=u_{0}(t)=\sin (x)+\frac{1}{4} x^{4}, \\
u(0, t)=g_{0}(t)=0, \quad u(1, t)=g_{1}(t)=e^{-t} \sin (1)+3 t+\frac{1}{4}, \\
u\left(x_{0}, t\right)=h(t)=e^{-t} \sin \left(\frac{1}{2}\right)+\frac{3}{4} t+\frac{1}{64},
\end{gathered}
$$

the inverse problem (2.1)-(2.5) has the unique solution given by

$$
\begin{gathered}
u(x, t)=e^{-t} \sin (x)+3 t x^{2}+\frac{1}{4} x^{4}, \\
f(t)=-6 t
\end{gathered}
$$


Table 1: Maximum absolute errors of $f_{n}$ and $u_{n}$ with exact input data for Example 4.1.

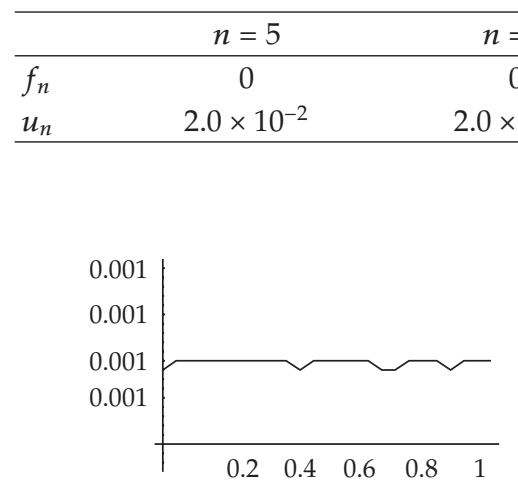

(a)

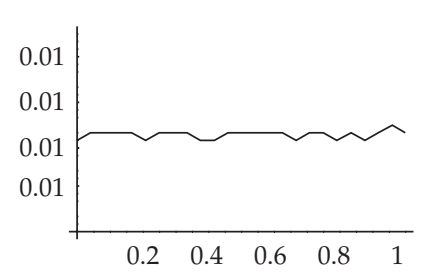

(b)

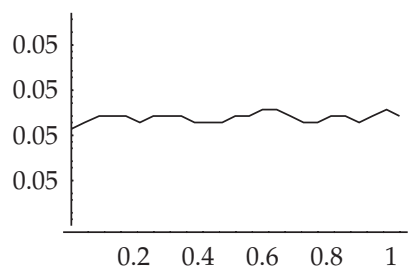

(c)

Figure 1: The figure of relative error $\left|\left(f-f_{5}\right) / f\right|$ with noise $\sigma=0.1 \%, 1 \%, 5 \%$, respectively, for Example 4.1.

Beginning with

$$
v_{0}(x, t)=v(x, 0)=\sin (x)+\frac{1}{4} x^{4},
$$

according to (3.9), one can obtain the successive approximations $v_{n}(x, t)$ of $v(x, t)$.

From (3.10), one can obtain the $n$-order approximation of $f(x)$ by

$$
f_{n}(t)=\left(g_{0}(t)-v_{n}(0, t)\right)^{\prime}
$$

and the $n$-order approximation of $u(x, t)$ by

$$
u_{n}(x, t)=v_{n}(x, t)+g_{0}(t)-v_{n}(0, t) .
$$

When the input dataware is exact, the maximum absolute errors of $f_{n}$ and $u_{n}$ are given in Table 1.

When the input dataware is noisy, with various levels of noise $\sigma=0.1 \%, \sigma=1 \%$, $\sigma=5 \%$, the relative errors of $f_{5}$ and $u_{5}$ are given in Figures 1 and 2 .

Example 4.2. With the input data

$$
\begin{gathered}
u(x, 0)=u_{0}(t)=x^{2}, \\
u(0, t)=g_{0}(t)=2 t+\sin (2 \pi t), \quad u(1, t)=g_{1}(t)=1+2 t+\sin (2 \pi t), \\
u\left(x_{0}, t\right)=h(t)=\frac{1}{4}+2 t+\sin (2 \pi t),
\end{gathered}
$$




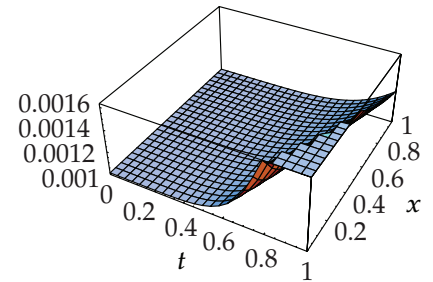

(a)

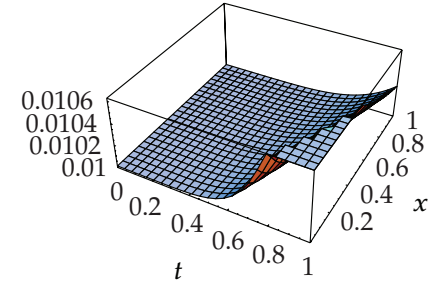

(b)

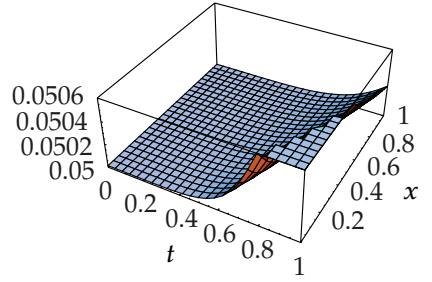

(c)

Figure 2: The figure of relative error $\left|\left(u-u_{5}\right) / u\right|$ with noise $\sigma=0.1 \%, 1 \%, 5 \%$, respectively, for Example 4.1.

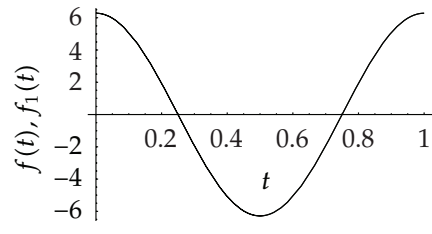

(a)

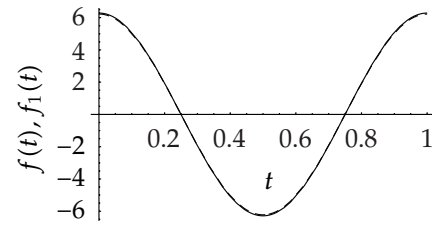

(b)

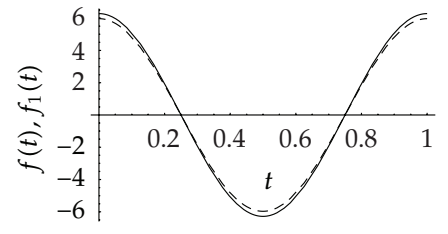

(c)

Figure 3: Figures of $f(t)$ and $f_{1}(t)$ with noise $\sigma=0.1 \%, 1 \%, 5 \%$, respectively, for Example 4.2 (the real line is $f(t)$ and the dashed line is $f_{1}(t)$ ).

the inverse problem (2.1)-(2.5) has the unique solution given by

$$
\begin{gathered}
u(x, t)=x^{2}+2 t+\sin (2 \pi t), \\
f(t)=2 \pi \cos (2 \pi t) .
\end{gathered}
$$

Beginning with

$$
v_{0}(x, t)=v(x, 0)=x^{2},
$$

according to (3.9), one can obtain the successive approximations $v_{n}(x, t)$ of $v(x, t)$.

From (3.10), one can obtain the $n$-order approximation of $f(x)$ by

$$
f_{n}(t)=\left(g_{0}(t)-v_{n}(0, t)\right)^{\prime}
$$

and the $n$-order approximation of $u(x, t)$ by

$$
u_{n}(x, t)=v_{n}(x, t)+g_{0}(t)-v_{n}(0, t) .
$$

When the input dataware is exact, the maximum absolute errors of $f_{n}$ and $u_{n}$ are zero. When the input dataware is noisy, with various levels of noise $\sigma=0.1 \%, \sigma=1 \%$, $\sigma=5 \%$, the relative errors of $f_{5}$ and $u_{5}$ are given in Figures 3 and 4 . 


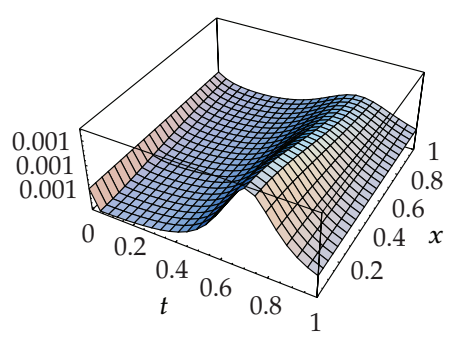

(a)

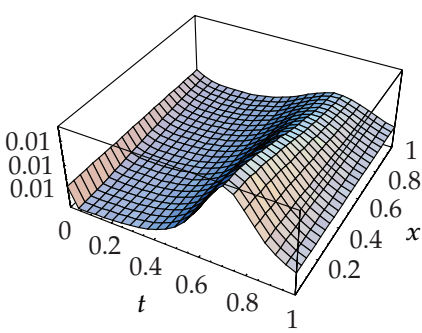

(b)

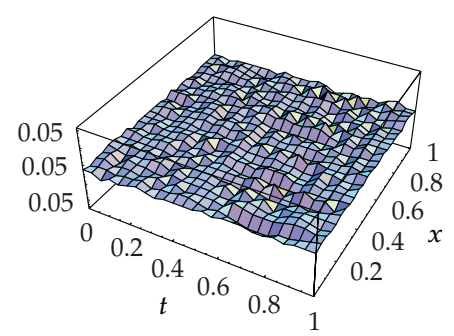

(c)

Figure 4: The figure of relative error $\left|\left(u-u_{1}\right) / u\right|$ with noise $\sigma=0.1 \%, 1 \%, 5 \%$, respectively, for Example 4.2.

Remark 4.3. From the previous two numerical examples, it can be seen that the numerical results are quite satisfactory. When the input data are exact, the numerical solutions obtained using our method are of high degree of accuracy. Even with the noise level of input data up to $\sigma=5 \%$, the numerical solutions are still in good agreement with the exact solutions.

\section{Conclusion}

In this paper, He's variational iteration method was employed successfully for solving the inverse heat source problems. This method solves the problem without any discretization of the variables. Therefore, it is not affected by computation round-off errors, and one is not faced with necessity of large computer memory and time. The numerical results show that the VIM is an accurate and reliable numerical technique for the solution of the inverse timedependent heat source problem.

\section{Acknowledgments}

The work of the author is supported by the Special Funds of the National Natural Science Foundation of China (Grant no. 11141003). The author would like to thank the referees for constructive suggestions and comments.

\section{References}

[1] J. R. Cannon and P. DuChateau, "Structural identification of an unknown source term in a heat equation," Inverse Problems, vol. 14, no. 3, pp. 535-551, 1998.

[2] A. G. Fatullayev, "Numerical solution of the inverse problem of determining an unknown source term in a two-dimensional heat equation," Applied Mathematics and Computation, vol. 152, no. 3, pp. 659-666, 2004.

[3] E. G. Savateev, "On problems of determining the source function in a parabolic equation," Journal of Inverse and Ill-Posed Problems, vol. 3, no. 1, pp. 83-102, 1995.

[4] D. D. Trong, N. T. Long, and P. N. D. Alain, "Nonhomogeneous heat equation: identification and regularization for the inhomogeneous term," Journal of Mathematical Analysis and Applications, vol. 312, no. 1, pp. 93-104, 2005.

[5] A. Farcas and D. Lesnic, "The boundary-element method for the determination of a heat source dependent on one variable," Journal of Engineering Mathematics, vol. 54, no. 4, pp. 375-388, 2006.

[6] L. Ling, M. Yamamoto, Y. C. Hon, and T. Takeuchi, "Identification of source locations in twodimensional heat equations," Inverse Problems, vol. 22, no. 4, pp. 1289-1305, 2006. 
[7] Zh. Yi and D. A. Murio, "Source term identification in 1-D IHCP," Computers \& Mathematics with Applications, vol. 47, no. 12, pp. 1921-1933, 2004.

[8] L. Yan, C.-L. Fu, and F.-L. Yang, "The method of fundamental solutions for the inverse heat source problem," Engineering Analysis with Boundary Elements, vol. 32, no. 3, pp. 216-222, 2008.

[9] M. Dehghan and M. Tatari, "Identifying an unknown function in a parabolic equation with overspecified data via He's variational iteration method," Chaos, Solitons E Fractals, vol. 36, no. 1, pp. 157-166, 2008.

[10] A. Golayoglu Fatullayev and S. Cula, "An iterative procedure for determining an unknown spacewise-dependent coefficient in a parabolic equation," Applied Mathematics Letters, vol. 22, no. 7, pp. 1033-1037, 2009.

[11] A. G. Fatullayev, "Numerical method of identification of an unknown source term in a heat equation," Mathematical Problems in Engineering. Theory, Methods and Applications, vol. 8, no. 2, pp. 161-168, 2002.

[12] A. G. Fatullayev, "Numerical solution of the inverse problem of determining an unknown source term in a heat equation," Mathematics and Computers in Simulation, vol. 58, no. 3, pp. 247-253, 2002.

[13] J.-H. He, "Variational iteration method-a kind of non-linear analytical technique: some examples," International Journal of Non-Linear Mechanics, vol. 34, no. 4, pp. 699-708, 1999.

[14] J.-H. He, "Some asymptotic methods for strongly nonlinear equations," International Journal of Modern Physics B, vol. 20, no. 10, pp. 1141-1199, 2006.

[15] J. Lu, "Variational iteration method for solving two-point boundary value problems," Journal of Computational and Applied Mathematics, vol. 207, no. 1, pp. 92-95, 2007.

[16] E. Yusufoglu, "Variational iteration method for construction of some compact and noncompact structures of Klein-Gordon equations," International Journal of Nonlinear Sciences and Numerical Simulation, vol. 8, no. 2, pp. 153-158, 2007.

[17] H. Tari, D. D. Ganji, and M. Rostamian, "Approximate solutions of K (2,2), KdV and modified KdV equations by variational iteration method, homotopy perturbation method and homotopy analysis method," International Journal of Nonlinear Sciences and Numerical Simulation, vol. 8, no. 2, pp. 203-210, 2007.

[18] D. D. Ganji and A. Sadighi, "Application of He's homotopy-perturbation method to nonlinear coupled systems of reaction-diffusion equations," International Journal of Nonlinear Sciences and Numerical Simulation, vol. 7, no. 4, pp. 411-418, 2006.

[19] Z. M. Odibat and S. Momani, "Application of variational iteration method to nonlinear differential equations of fractional order," International Journal of Nonlinear Sciences and Numerical Simulation, vol. 7, no. 1, pp. 27-34, 2006.

[20] N. Bildik and A. Konuralp, "The use of variational iteration method, differential transform method and adomian decomposition method for solving different types of nonlinear partial differential equations," International Journal of Nonlinear Sciences and Numerical Simulation, vol. 7, no. 1, pp. 65$70,2006$.

[21] F. Geng and Y. Lin, "Numerical solution of a system of fourth order boundary value problems using variational iteration method," Applied Mathematics and Computation, vol. 200, no. 1, pp. 231-241, 2008.

[22] J. Biazar and H. Ghazvini, "He's variational iteration method for solving linear and non-linear systems of ordinary differential equations," Applied Mathematics and Computation, vol. 191, no. 1, pp. 287-297, 2007.

[23] M. Dehghan and A. Saadatmandi, "Variational iteration method for solving the wave equation subject to an integral conservation condition," Chaos, Solitons \& Fractals, vol. 41, no. 3, pp. 1448-1453, 2009.

[24] S. M. Goh, A. I. M. Ismail, M. S. M. Noorani, and I. Hashim, "Dynamics of the hantavirus infection through variational iteration method," Nonlinear Analysis, vol. 10, no. 4, pp. 2171-2176, 2009.

[25] A.-M. Wazwaz, "A study on linear and nonlinear Schrodinger equations by the variational iteration method," Chaos, Solitons and Fractals, vol. 37, no. 4, pp. 1136-1142, 2008.

[26] A.-M. Wazwaz, "The variational iteration method for analytic treatment of linear and nonlinear ODEs," Applied Mathematics and Computation, vol. 212, no. 1, pp. 120-134, 2009. 


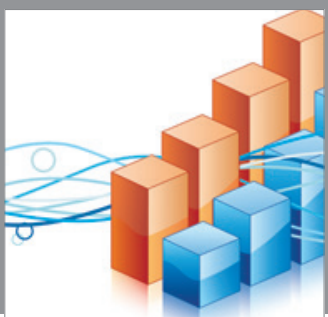

Advances in

Operations Research

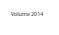

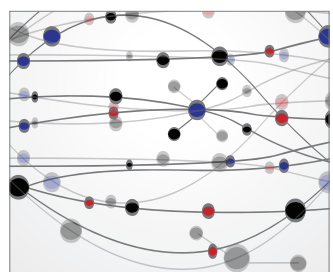

\section{The Scientific} World Journal
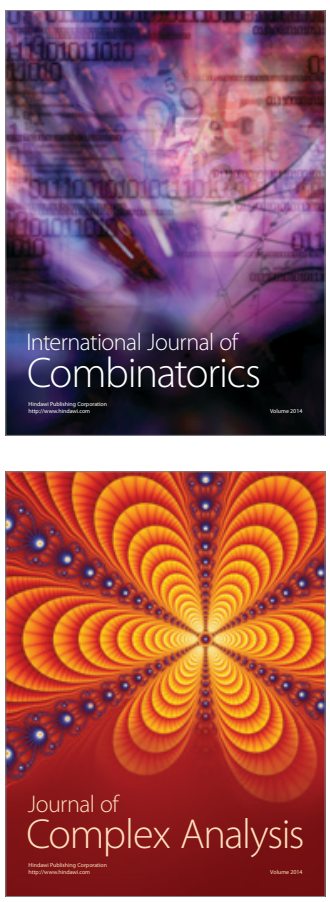

International Journal of

Mathematics and

Mathematical

Sciences
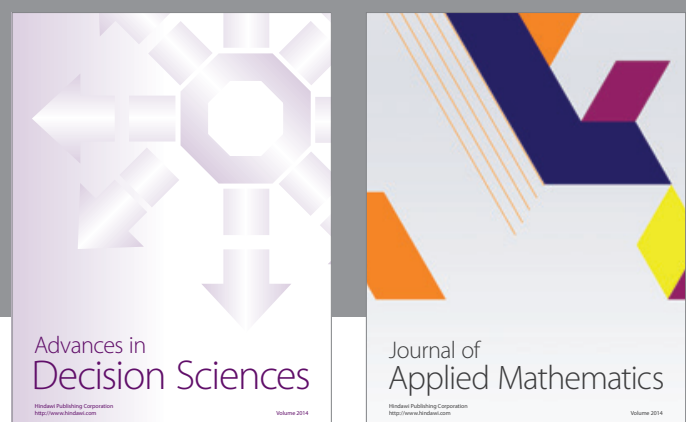

Journal of

Applied Mathematics
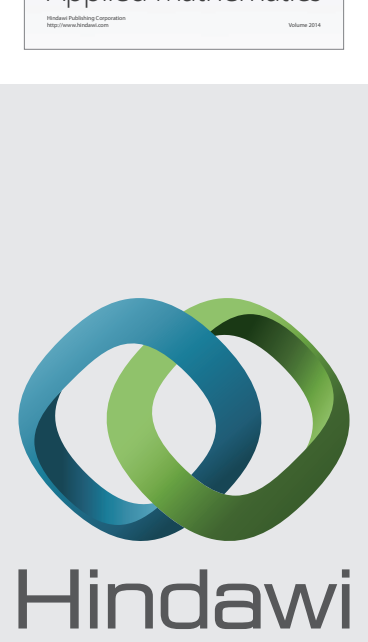

Submit your manuscripts at http://www.hindawi.com
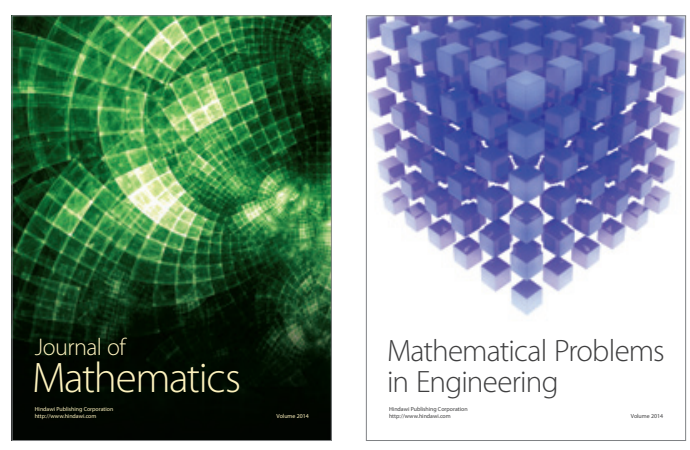

Mathematical Problems in Engineering
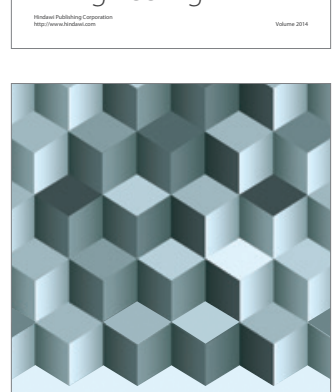

Journal of

Function Spaces
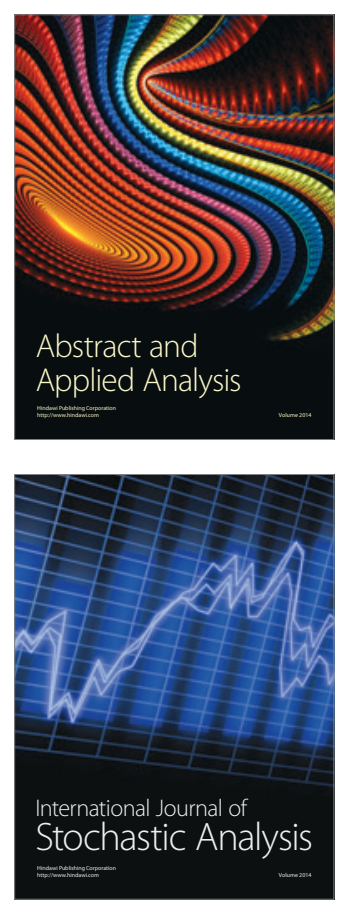

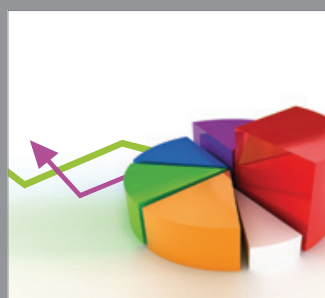

ournal of

Probability and Statistics

Promensencen
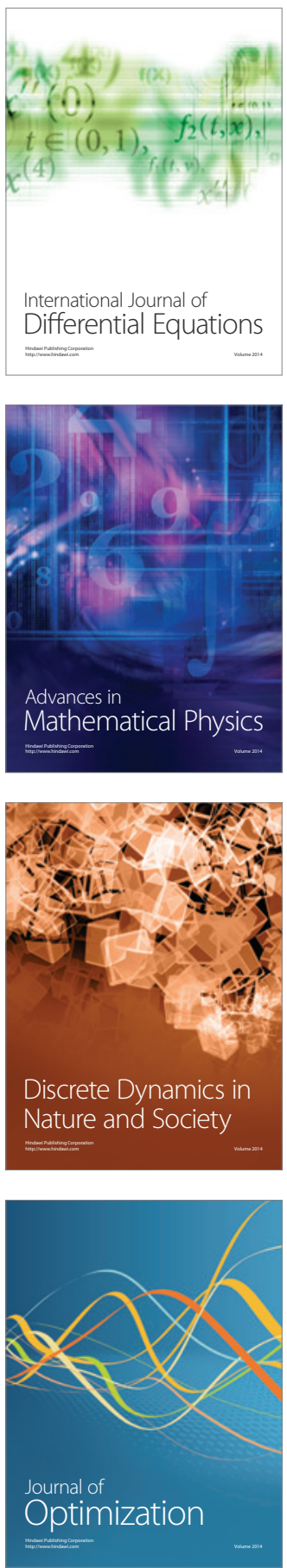